\title{
ALGUNAS CONSIDERACIONES SOBRE LAS RELACIONES INTRA- FAMILIARES Y LAS REDES DE APOYO EN LA CHINA ACTUAL
}

\author{
FLORA BOTTON BEJA \\ El Colegio de México
}

La familia, base de la sociedad china, se mantuvo con pocos cambios hasta el triunfo de la revolución, en 1949. Excepto por las clases más ilustradas de algunas zonas urbanas, era patriarcal y jerárquica, y sus miembros más oprimidos eran los jóvenes y sobre todo las mujeres. El patriarca tenía un poder casi absoluto sobre los demás miembros de la familia, administraba todos sus bienes y decidía el matrimonio de los hijos.

La familia tradicional, cuya estructura contribuyó a la estabilidad y la permanencia de actitudes confucianas, y cuyo ideal era la armonía, no estuvo exenta de conflictos entre los intereses del grupo familiar y la felicidad personal, entre el orden jerárquico y la igualdad social, así como la de género; de hecho, había tensiones profundas entre el ideal y la realidad en las relaciones sociales en China. ${ }^{1}$

Después del triunfo de la revolución de 1949, la familia tradicional fue uno de los blancos más atacados por el régimen posrevolucionario y casi no hubo ámbito en las transformaciones que llevaba a cabo el Partido Comunista que no tuviera alguna relación con la familia; por eso, a la par que las leyes sobre la reforma agraria, el reparto de tierras y el derecho al trabajo, una de las leyes que mayor efecto tuvieron para transformar a la familia fue la Ley de Matrimonio, promulgada en

Este artículo fue recibido por la dirección de la revista el 12 de enero de 2015 y aceptado para su publicación el 13 de marzo de 2015.

${ }^{1}$ Véase Flora Botton Beja y Romer Cornejo Bustamante, Bajo un mismo techo: la familia tradicional en China y su crisis, México, El Colegio de México, 1993. 
1950. La Ley de Matrimonio de 1950 declaraba la igualdad de derechos sin distinción de sexo, no reconocía a un "jefe de familia" y prohibía los matrimonios arreglados, la interferencia en el matrimonio de las viudas, el compromiso de niños y el pago de dote. Se hacía obligatoria la monogamia y se permitía el divorcio en igualdad de condiciones.

Las reformas fomentadas por Deng Xiaoping después de la muerte de Mao, que marcaron el final de la revolución cultural y la salida del poder del grupo radical, han tenido una estrecha relación con la familia. En el campo, con la paulatina desaparición de las comunas a partir de 1979, se adoptó el sistema de responsabilidad familiar. Este sistema provocó un gran aumento de la producción y el mejoramiento del nivel de vida de los campesinos; sin embargo, el funcionamiento de la familia como unidad básica de propiedad, producción, distribución y consumo suscitó cambios en las relaciones entre sus miembros. La necesidad de mano de obra masculina dentro del seno de la familia y la reaparición de ceremonias religiosas y cultos tradicionales agudizaron la necesidad de tener hijos varones. Por otra parte, la posición de las mujeres, a quienes les asignaron tareas no remuneradas de reproducción o de producción casera, sufrió un gran deterioro. ${ }^{2}$

Justamente, en el momento en que aumentaba la presión para tener hijos varones, el Estado propugnó una política de población en la que se limitaba a las parejas a tener un solo hijo. ${ }^{3} \mathrm{El}$ instrumento jurídico que acentuó la consolidación del grupo familiar como unidad económica, con deberes bien definidos para cada uno de los miembros, fue la nueva Ley de Matrimonio, de 1980. Se hicieron modificaciones en cuanto a la edad legal para contraer matrimonio (se aumentó de 20 a 22 años para los hombres y de 18 a 20 para las mujeres), se definió la responsabilidad legal de ambos cónyuges en el control de la natalidad, se estableció una mayor flexibilidad para obtener el divorcio y se puso énfasis en el cuidado de los ancianos y la edu-

${ }^{2}$ Véase Flora Botton Beja, "Familia y cambio social", Revista de Occidente, núm. 172, septiembre de 1995, pp. 51-64.

${ }^{3}$ Véase Flora Botton Beja y Romer Cornejo, "La política de un solo hijo en China”, Estudios Demográficos y Urbanos, vol. 4, núm. 2 (11), mayo-agosto de 1989, pp. 343-376. 
cación de los hijos, con lo que se descargaron una vez más sobre la familia funciones sociales tradicionales. En ambas Leyes de Matrimonio hay una clara intención de cambiar la estructura de la sociedad dándole al Estado el control de los medios de producción, distribución de los puestos de trabajo, educación, etcétera. ${ }^{4}$

Hasta fines de la década de 1980, la transformación de la familia en China no se había dado mucho más allá de los patrones que habían fijado los cambios en la economía y la legislación después del triunfo de la revolución de 1949. En una investigación que realizamos en 1987 en seis ciudades chinas, dos de mis colegas y yo tuvimos la oportunidad de aplicar cuestionarios y de entrevistarnos con varias personas, quienes hablaron sobre su situación familiar. ${ }^{5}$ Nuestros entrevistados, en general, se manifestaban satisfechos con su pareja, insistían sobre la importancia del respeto mutuo, mencionaban poco el amor, no aprobaban el divorcio salvo en situaciones extremas, y en todos los casos atribuían los problemas entre las parejas a diferencias sobre la educación de los hijos y la ayuda económica a algún familiar de la pareja. Es preciso señalar que en la mayoría de los casos, los entrevistados manifestaron haber conocido a su pareja a través de los buenos oficios de terceros, y que sus relaciones no eran necesariamente producto de un enamoramiento. El gran problema para todos era el de la vivienda y el espacio del cual disponían. En nuestra muestra, sólo aproximadamente $4 \%$ tenían casa propia; al resto, en su mayoría, la unidad de trabajo les había asignado la vivienda y frecuentemente debían compartir con otras familias la cocina y otras áreas. ${ }^{6}$

El cambio en la sociedad china en la década de 1980 fue frenado por el incidente de la Plaza Tian An'men, que retrasó toda liberalización, salvo la económica; sin embargo, los cambios

${ }^{4}$ Véase Flora Botton Beja y Romer Cornejo, "Cambio y tradición en la familia china contemporánea”, Revista de la Universidad Nacional Autónoma de México, vol. XLV, núm. 474, julio de 1990, pp. 33-37.

${ }^{5}$ Investigación realizada por Flora Botton, Romer Cornejo y Liljana Arsovska en 1987 en las siguientes ciudades: Beijing, Harbin, Xi’an, Nanning, Jinan y Shenzhen.

${ }^{6}$ Véase Flora Botton Beja y Romer Cornejo, "Vivienda y familia en la China urbana contemporánea”, Estudios Demográficos y Urbanos, vol. 6, núm. 1 (16), eneroabril de 1991, pp. 187-207. 
económicos tuvieron a la larga consecuencias en otros ámbitos. En la nueva sociedad china, aun las instituciones tradicionalmente más sólidas eran cuestionadas y sufrían cambios; entre ellas, la familia, que había mantenido algunos principios básicos como la universalidad del matrimonio, la necesidad de tener hijos (aunque sea un solo hijo) y la unidad económica de los miembros.

En realidad, la verdadera transformación de la sociedad china se debe a reformas mucho más recientes, que se produjeron a principios, pero sobre todo a mediados de la década de 1990. En primer lugar, el desmantelamiento del predominio de la economía estatal y la venta de empresas estatales provocaron y permitieron el surgimiento de la economía privada, que en algunos casos se tradujo en grandes consorcios pero que las más de las veces tomó la forma de pequeños negocios o de iniciativas personales o familiares, lo que significó un gran aumento del ingreso per cápita, el cual subió de 3800 dólares en 1999, a 8500 dólares en 2011.

Las consecuencias más obvias en las relaciones familiares en esta situación son una mayor libertad de los jóvenes —sobre todo en las ciudades- para elegir una pareja, una disminución en la urgencia de tener hijos, relaciones de poder más igualitarias entre hombres y mujeres, pero menores niveles de tolerancia en la convivencia, lo que resulta en el aumento de los divorcios. Además, las presiones que impone una nueva economía de mayor consumismo y el deseo de satisfacer necesidades materiales han incidido en casos de violencia intrafamiliar. En el campo, uno de los mayores problemas es la desproporción entre hombres y mujeres, que hace más difícil para los hombres pobres conseguir esposa y fomenta prácticas ilegales como la venta de mujeres o el rapto para obtener una pareja. Las transformaciones económicas aunadas a una mayor apertura política tuvieron como consecuencia una transformación en las relaciones familiares y matrimoniales, que obligó al gobierno a proceder a una revisión de la Ley de Matrimonio de 1980. La ley revisada fue publicada en el año 2000 y promulgada en 2001. Esta ley es una enmienda y ampliación de la ley de 1980, y considera situaciones más complejas en las relaciones intrafamiliares. En un intento por proteger a las mujeres, se pre- 
vió el problema de las relaciones extramatrimoniales, consecuencia de la nueva prosperidad económica; se ampliaron y especificaron causales de divorcio, que incluyen maltrato, abandono, infidelidad, bigamia, etcétera, y se agregaron cláusulas sobre patria potestad, manutención y responsabilidad económica hacia los hijos y los padres ancianos. En esta nueva versión de la Ley de Matrimonio, que pretende responder a los cambios que ha sufrido la sociedad china en los últimos años, se percibe, al mismo tiempo, un esfuerzo para poner límites a ciertos cambios que, según la apreciación de las autoridades, se oponen a los valores familiares. Hay una conciencia creciente de que existen personas vulnerables que deben ser protegidas: las mujeres, los niños y los ancianos. La propiedad privada, poco importante en la China de antes, se vuelve un problema que da lugar a disputas y litigios; sin embargo, aun con las modificaciones, la nueva ley es un esfuerzo más por afianzar valores tradicionales que consideran a la familia como el pilar que siempre sostuvo a la sociedad china y de ninguna manera anticipa formas alternativas de convivencia, frecuentes en el mundo actual. En general, a pesar de los cambios y de las voces de alarma, la institución de la familia no fue puesta en duda por la mayoría de la población y el matrimonio aún constituye la manera más aceptable para formar una pareja.?

Estudiar las relaciones intrafamiliares y las redes de apoyo en la China actual nos da la clave para evaluar los cambios en una sociedad tradicionalmente dominada por estas relaciones; es también importante considerar que los cambios no se dieron, en un principio, como consecuencia natural de la modernización y evolución de la sociedad, sino que fueron acelerados por leyes promulgadas por el Estado y una supervisión para garantizar su aplicación. Si bien a partir de mediados de la década de 1980 los cambios fueron generados por influencia de reformas económicas y políticas, podemos decir que una vez más la injerencia del Estado fue primordial. En la actualidad, cuando los cambios dentro de la sociedad china tienen una evolución más autónoma, chasta qué punto los patrones tradicionales en las re-

7 Véase Flora Botton Beja, "Cambio social y cultura”, en Romer Cornejo (coord.), China: radiografía de una potencia en ascenso, México, El Colegio de México, 2008, pp. 121-206. 
laciones intrafamiliares se han modificado o en qué medida prevalecen aún?

La investigación sobre la familia en China estuvo limitada por las circunstancias políticas que prevalecieron en ese país después del triunfo de la revolución en 1949; aun en épocas anteriores hubo pocas investigaciones debido al desarrollo incipiente de la sociología y la antropología. Fueron pioneros en estos estudios Olga Lang y Fei Xiaotong. ${ }^{8}$ En los primeros veinte años de la República Popular China, las investigaciones sobre la familia se hacían mayormente a través de entrevistas con refugiados en Hong Kong;' además, aparecieron estudios sobre la situación de las mujeres en China realizados por estudiosas francesas, inglesas y canadienses. ${ }^{10} \mathrm{La}$ dificultad para hacer trabajo de campo volvía necesario el uso de información de diarios y revistas chinos, y los informes de agencias chinas, como Xinhua, y occidentales de monitoreo de la prensa y la radio de China. ${ }^{11}$ Ya en la década de 1980, aparecieron algunas investigaciones realizadas en China - como la que dirigió Xu Anqien las que se utilizaron métodos más modernos. ${ }^{12} \mathrm{~A}$ fines de la década de 1980 y principios de la de 1990 se popularizaron estudios sobre demografía, género y sexualidad realizados por investigadores occidentales, y también chinos, algunos educados en universidades occidentales. ${ }^{13} \mathrm{Al}$ mismo tiempo, se popularizó, en periódicos y revistas, el uso de encuestas sobre una gran variedad de temas que atañen a la familia y a la relación entre sus miembros.

${ }^{8}$ Olga Lang, Chinese Family and Society, New Haven, Yale University Press, 1946, p. X. Olga Lang hizo trabajo de campo en China entre 1935-1937. Fei Xiaotong fue un antropólogo y sociólogo pionero en señalar la importancia de las relaciones en China a través de redes sociales de apoyo.

${ }^{9}$ Martin King Whyte y William L. Parish, Village and Family in Contemporary China, Chicago, The University of Chicago Press, 1978, y William L. Parish y Martin King Whyte, Urban Life in Contemporary China, Chicago, The University of Chicago Press, 1984.

${ }^{10}$ Simone de Beauvoir, Janet Salaf, Delia Davin, Elizabeth Croll, etcétera.

${ }^{11}$ China News Analysis, China News and World Report, BBC bulletins, Xinbua Neres Bulletin.

${ }_{12}$ Zhongguo chengshi jiating: Wu chengshi jiating diaocha baogao he ziliao (La familia en cinco ciudades chinas: colección de archivos e informes de la investigación sobre la familia en cinco ciudades), Jinan, Shandong Renmin Chubanshe, 1985.

${ }^{13}$ Pan Suiming Liu Xiaojiang, Liu Deling, Peng Xizhe. 
Esta tendencia se acentuó con la mayor apertura de China y han proliferado las investigaciones. Al examinar algunos de los estudios hechos a partir de mediados de la década de 1990 y después del año 2000 sobre relaciones intrafamiliares y grupos de apoyo, vemos una gran variedad de opiniones y conclusiones. Las investigaciones abarcan áreas tanto urbanas como rurales y pueden ser muestras pequeñas o más amplias, basadas en censos nacionales, a veces apoyadas en encuestas o en entrevistas. No se pretende hacer un análisis cuantitativo de los resultados de estas investigaciones; más bien, se presentarán algunas de las propuestas que ofrecen los investigadores. No todos ellos están de acuerdo sobre ciertos aspectos de las relaciones intrafamiliares y las redes de apoyo, pero todos se interesan en identificar los cambios o la permanencia de ciertas características de la familia tradicional. ${ }^{14}$

Cuando discuten el tema de la influencia de las reformas económicas sobre la familia, especialmente la familia rural, los estudiosos proponen dos teorías: una que ve un retorno a la tradición ahora que la familia vuelve a ser la unidad de producción, y otra que sostiene que las nuevas oportunidades de empleo "no agrícola" hacen que la familia se aleje de la estructura y los valores tradicionales, sobre todo ahora que se ha vuelto menos estricta la regla de residencia y ha aumentado la movilidad. ${ }^{15}$ Algunos investigadores afirman que tanto las Leyes de Matrimonio de 1950 y 1980 como los ataques al culto de los ancestros y el desmantelamiento de organizaciones de linaje han minado la autoridad paterna. Asimismo, el control del Estado de los medios de producción y la distribución de puestos de trabajo y educación ha disminuido la cohesión familiar. ${ }^{16}$ Para Martin Whyte, puede apreciarse la diferencia de actitud hacia la

${ }^{14}$ Véase tabla en el Anexo.

${ }^{15}$ Yan Yunxiang, "The Triumph of Conjugality: Structural Transformation of Family Relations in a Chinese Village”, Ethnology, vol. 36, núm. 3, 1997, p. 203; Martin King Whyte, "The Fate of Filial Obligations in Urban China”, The China Journal, núm. 38, julio de 1997, pp. 1-31; Xu Anqi et al., Chinese Family Strengths and Resiliency, Documento núm. 53, Lincoln, Faculty Publications, Department of Child, Youth and Family Studies, 2007, pp. 143-164.

${ }^{16}$ Nancy E. Riley, "Interwoven Lives: Parents, Marriage, and Guanxi in China", Journal of Marriage and Family, vol. 56, núm. 4, noviembre de 1994, pp. 792-793; Yan Yunxiang, "The Triumph of Conjugality”, op. cit., p. 207. 
familia a través del deterioro de la piedad filial, que considera la obligación hacia la familia como la máxima virtud confuciana y la más importante en las relaciones humanas. Este deterioro es el resultado de muchos años de propaganda comunista, que privilegia la lealtad hacia el partido, la nación y Mao sobre la familia. Al mismo tiempo, el control del Estado socialista sobre las escuelas, lugares de trabajo y hasta la residencia, hizo perder mucho del control que los padres tenían sobre los hijos. ${ }^{17}$ Además, los cambios en la economía china han ayudado a crear una sociedad más educada, más "moderna”, y "la transformación de una sociedad agraria hacia una sociedad industrial tiene un efecto predecible sobre patrones de la vida familiar". ${ }^{18}$ Convencido de que los cambios en el ámbito rural son indicativos de un cambio general dentro de la sociedad china, Yan Yunxiang estudió durante once años (1989-1991) la aldea de Xiajia, en Heilonjiang, en el noreste de China; examinó estadísticas y se entrevistó con mujeres jóvenes y matrimonios durante ese tiempo y hasta 1999. ${ }^{19}$ Lo que observa Yan es que hay una transformación estructural de la vida doméstica, tanto en ideología como en práctica, y se percibe un cambio de las relaciones de padre a hijo antes dominantes, por una tendencia a privilegiar lo que llama la "conyugalidad" sobre el patriarcado, cuando la relación de la pareja prevalece sobre la relación con los padres. ${ }^{20}$ En varios casos de conflicto, cuando sería normal que el hijo apoyara a sus padres, ahora apoya a la esposa. Esto lo atribuye, entre otras cosas, a las nuevas oportunidades para trabajar en las ciudades, consecuencia del relajamiento de las leyes de residencia que permiten una mayor movilidad. Desde mediados de la década de 1980 hasta 1993, más de 100 millones de campesinos emigraron a las ciudades. ${ }^{21}$ Otro de los cambios importantes es la corresidencia. Anteriormente, en el campo, una familia independiente se constituía por la división de la fa-

${ }^{17}$ Whyte, “The Fate of Filial Obligations in Urban China”, op. cit., p. 2.

${ }^{18}$ Ibid., p. 3.

${ }^{19}$ Yan Yunxiang, "The Triumph of Conjugality", op. cit.; y "Girl Power: Young Women and The Waning of Patriarchy in Rural North China”, Ethnology, vol. 45, núm. 2, primavera de 2006, pp. 105-123.

${ }^{20}$ Yan Yunxiang, “The Triumph of Conjugality...”, op. cit., p. 209.

${ }^{21}$ Yan Yunxiang, "Girl Power", op. cit., p. 117. 
milia extendida después de muchos años de residencia común, tal vez a la muerte del patriarca. ${ }^{22}$ Sin embargo, hacia 1994 , $40 \%$ de los recién casados vivían independientemente. ${ }^{23}$ Se ha discutido mucho hasta qué punto el fin de la colectivización y el regreso a la producción familiar alentaron la asunción de viejos patrones de estructura familiar. Durante la colectivización, la familia dejó de ser la unidad de producción y el liderazgo paterno fue reemplazado por el liderazgo de los cuadros de la aldea. Este nuevo orden de producción tuvo como resultado una disminución de la autoridad paterna: $;^{24}$ sin embargo, Yan señala que a pesar de que fue restaurada la producción familiar, no sucedió así con todo el modo de organización social, puesto que la economía familiar está regida por políticas del Estado y exigencias del mercado. ${ }^{25}$

$\mathrm{Xu}$ Anqi, por otro lado, tiene una actitud más conservadora en cuanto a la supervivencia de valores familiares tradicionales y ve la familia actual como la base de apoyo de sus miembros. También señala que hasta fines de la década de 1990, la escasez de vivienda obligaba a muchos hijos casados a seguir viviendo en la casa paterna, con lo que debían acatar la autoridad de los miembros mayores de la familia. Es también importante la sobrevivencia de actitudes confucianas, que ponen énfasis sobre el deber hacia los padres, la armonía y el bien común, y hacen ver a la familia como centro de apoyo emocional y material en épocas de crisis. ${ }^{26}$

A pesar de las opiniones divergentes existe consenso entre los estudiosos de que los lazos entre los miembros de la familia permanecen vivos y fuertes. La universalidad del matrimonio está todavía vigente y es mal vista una persona soltera, sobre todo si es mujer. ${ }^{27}$ Los padres aún tienen injerencia en la decisión de matrimonio, porque los hijos generalmente viven en la casa paterna hasta casarse $y$, hasta las nuevas reformas de

${ }^{22}$ Yan Yunxiang, “The Triumph of Conjugality”, op. cit., p. 198.

${ }^{23}$ Yan Yunxiang, "Girl Power", op. cit., p. 112.

${ }^{24}$ Ibid., p. 115.

${ }^{25}$ Yan Yunxiang, “The Triumph of Conjugality”, op. cit., pp. 206-207.

${ }^{26}$ Xu Anqi et al., Chinese Family Strengths and Resiliency, op. cit., pp. 154-155.

${ }^{27}$ Riley, "Interwoven Lives: Parents, Marriage, and Guanxi in China", op. cit., p. 721 . 
vivienda en la década de 1990, porque muchos jóvenes matrimonios tenían que vivir con los padres. Esta convivencia exige que de alguna manera los padres se involucren en la vida social de sus hijos y tengan opinión sobre sus posibles parejas. ${ }^{28}$ No obstante el cambio en el tamaño de la familia y actitudes más liberales de los jóvenes hacia el matrimonio y la familia, la falta de servicios sociales hace que ésta sea todavía responsable del cuidado de los niños y de su socialización, así como del apoyo a los ancianos. ${ }^{29}$ Desde las reformas económicas y como consecuencia de las estrictas políticas de población, en algunos aspectos los ancianos son más dependientes económicamente. ${ }^{30}$ En las áreas rurales, donde no hay todavía un sistema de seguro universal, los hijos tienen que mantener a los padres, pero aun en los centros urbanos donde los padres tienen una pensión es importante el apoyo de los hijos, sobre todo cuando surgen problemas de salud. ${ }^{31}$ Ahora, una pareja adulta puede tener a su cargo a un niño y a una pareja de ancianos y, con la posibilidad de que dos hijos únicos pueden tener dos hijos, en un futuro cercano, la pareja tendrá a su cargo dos hijos y cuatro ancianos. $^{32}$

En otros aspectos de las relaciones intrafamiliares, un estudio de fines de la década de 1990 realizado por Xu Anqi revela que tanto hombres como mujeres se decían en general satisfechos con sus matrimonios y atribuían la mayor parte de los conflictos a temas como la educación de los hijos, cuestiones de dinero y, a veces, la repartición de las tareas domésticas, pero la incidencia del divorcio, que va en aumento, muestra el deterio-

${ }^{28}$ Ibid., p. 798.

${ }^{29} \mathrm{Xu}$ Anqi et al., Chinese Family Strengths and Resiliency, op. cit., pp. 160-161.

${ }^{30}$ Zhan Heying Jenny y Rhonda J. V. Montgomery, "Gender and Elder Care in China: The Influence of Filial Piety and Structural Constraints", Gender and Society, vol. 17, núm. 2, abril de 2003, pp. 209-210.

${ }^{31}$ Riley, "Interwoven Lives: Parents, Marriage, and Guanxi in China", op. cit., p. 779; Flora Botton Beja, "¿Qué hacer con los viejos? El problema del envejecimiento en China”, Estudios de Asia y África, vol. XLVIII (2), núm. 148, mayo-agosto de 2012, pp. 219-236.

${ }^{32}$ Di Juxin y Emily Rosenbaum, "Caregiving System in Transition: An Illustration from Shanghai, China”, Population Research and Policy Revierw, vol. 13, núm. 1, marzo de 1994, p. 104; Chen Feinan, Susan E. Short y Barbara Entwisle, "The Impact of Grandparental Proximity on Maternal Childcare in China", Population Research and Policy Review, vol. 19, núm. 6, diciembre de 2000, p. 585. 
ro en los niveles de satisfacción y de tolerancia provocados por mayores expectativas de las parejas. ${ }^{33}$ En cuanto a las relaciones entre padres e hijos pueden observarse, en teoría, todavía los patrones tradicionales del padre severo y disciplinario y de la madre indulgente, aunque las madres chinas pueden ser a veces más exigentes que los padres. ${ }^{34}$

Si los estragos de la revolución cultural —cuando hubo hijos que denunciaron a sus padres y muchas familias fueron separadas- han sido superados, en la actualidad hay otros problemas. Hasta fines de la década de 1980 prevalecía el patrón de los abuelos que ayudaban al cuidado de los niños y apoyaban en las tareas domésticas y, a su vez, el de los hijos que ayudaban a sus padres. Ahora, por un lado, han cambiado las reglas de cohabitación y, por ello, también las reglas de los cuidados; tanto en la ciudad como en el campo hay más familias nucleares y, si pueden permitírselo, tanto los viejos como los jóvenes prefieren vivir solos. Aun así, no se ha roto la posibilidad del apoyo mutuo y no es raro recurrir a la familia en caso de necesidad.

Un cambio reconocido por varios estudiosos es el que se ha dado en los patrones sexuales. La sociedad china se ha caracterizado por ser conservadora en cuanto a la sexualidad y, con el advenimiento de la República Popular, la "moral socialista" siguió la misma tendencia; sin embargo, en la actualidad, la actividad sexual premarital es ya algo común. Según estudios, $81 \%$ de las parejas que se casaron después de 1979 habían tenido relaciones sexuales antes del matrimonio. ${ }^{35}$ En otra muestra de estudiantes en Shanghái, 62\% aceptaron haber tenido relaciones sexuales con su pareja. ${ }^{36}$ No obstante, también es notorio

${ }^{33}$ Xu Anqi et al., Chinese Family Strengths and Resiliency, op. cit., pp. 148-151.

${ }^{34}$ Chan Darius K.-S., Theresa T.-T. Ng y Chin-Ming Hui, "Interpersonal Relationships in Rapidly Changing Chinese Societies", en Michael Harris Bond (ed.), Oxford Handbook of Chinese Psychology, Oxford-Nueva York, Oxford University Press, 2010, p. 525.

${ }^{35}$ S. M. Pan, "A Sex Revolution in Current China”, Journal of Psychology and Human Sexuality, vol. 6, núm. 2, 1993, pp. 1-14; Chan, Ng y Hui, "Interpersonal Relationships...”, op. cit., p. 520.

${ }^{36}$ S. Tang y J. Zuo, "Dating Attitudes and Behaviors of American and Chinese College Students”, Social Science Journal, vol. 37, núm. 1, 2000, pp. 67-78; Chan, Ng y Hui, "Interpersonal Relationships...”, op. cit., p. 520. 
que, en la mayoría de los casos, las relaciones premaritales son un preludio al matrimonio y no meras aventuras. Al mismo tiempo, los estudios demuestran que hay una gran ignorancia sobre la sexualidad y que, como consecuencia, han aumentado los abortos y las enfermedades de transmisión sexual.

En el pasado, las relaciones sociales y el culto religioso se regían por normas de parentesco que generaban agrupamientos de afines que reconocían a un ancestro común, a partir de lo cual se formaban linajes que participaban en ciertos rituales -el principal de los cuales era el culto a los ancestros- y que a menudo poseían propiedades comunales y un templo ancestral. Agrupaciones de varios linajes de un mismo apellido formaban clanes, que también se decían descendientes de un ancestro que podía ser un ser mitológico o un héroe de la antigüedad. Esta compleja red de linajes y de clanes, de alianzas y de apoyos, durante muchos siglos fue la base de la organización política en China y a la vez fuente de auxilio para las mismas familias.

En la actualidad, las redes de apoyo descansan en un sistema de relaciones interpersonales que se conoce como guanxi. Aunque no siempre, el guanxi puede tener origen en lazos de parentesco y generalmente se trata de intercambios entre iguales, donde unos tienen poder en un área y otros, en otra. En el pasado reciente, el guanxi era una manera de conseguir, fuera del mercado, bienes que eran escasos y servicios necesarios. Aún en la actualidad, el guanxi está presente en la vida cotidiana y es muy frecuente que los hijos dependan del guanxi de los padres a fin de resolver problemas de escolaridad o de trabajo. ${ }^{37}$

El guanxi es también importante para el autoempleo. Conseguir un empleo es más fácil que establecer un negocio propio, tanto a fin de obtener ayuda para financiarlo como para realizar los trámites necesarios exigidos por la burocracia. ${ }^{38}$ Donde es muy importante el guanxi es en la migración del campo a la ciudad. Las redes que conectan a migrantes con ex migrantes en áreas de origen y destino a través de lazos de parentesco, amistad y origen p. 791.

${ }^{37}$ Riley, "Interwoven Lives: Parents, Marriage, and Guanxi in China”, op. cit.,

${ }^{38}$ Zhang Junfu y Zhong Zhao, Social-Family Network and Self-Employment: Evidence from Temporary Rural-Urban Migrants in China, Documento de discusión núm. 5446, Bonn, Institute for the Study of Labor, enero de 2011, pp. 2-3. 
compartido de una comunidad son extremadamente importantes en China y peculiares dentro de un contexto mundial. ${ }^{39}$ Para los campesinos que migran es esencial este lazo para conseguir trabajo, y para los ya establecidos es una garantía de confianza y fidelidad de los que emplean. Sin embargo, a veces es un sistema que hace vulnerables a los trabajadores por la falta de contratos y por la discrecionalidad en los salarios.

Se calcula que hay 120 millones de migrantes del campo a las ciudades, de los cuales la mitad son mujeres que en las zonas económicas especiales llegan a ser 70\%. ${ }^{40}$ Mientras los migrantes varones prevalecen en la industria de la construcción, las mujeres son mayoría en las industrias textiles y de manufactura, además de los servicios. ${ }^{41}$ Estas mujeres, aunque por un lado están más protegidas, no tienen la seguridad y el respaldo de un contrato y pueden ser explotadas por su empleador, quien a veces las hace trabajar largas jornadas o no les paga puntualmente. Si bien las redes de apoyo facilitan la migración de mujeres, el ambiente en el que éstas se mueven es limitado y tienen pocas oportunidades de ampliar su círculo de conocidos y tener una vida propia, independiente, sin incurrir en críticas por no observar el recato exigido a su sexo. Como consecuencia, tienen menos posibilidades de progresar en sus empleos. ${ }^{42}$

La familia china está cambiando, tanto en el ámbito urbano como en el rural; sin embargo, sigue siendo la principal fuente de apoyo emocional y económico en épocas de crisis, $y$ hasta fines de la década de 1990 auxiliaba con la vivienda. También, al ser todavía escasos los servicios del Estado, ayuda con el cuidado de los niños y de los ancianos. En este último renglón es donde hay mayores problemas porque a pesar de que la piedad filial es aún ahora un valor apreciado en China, años de insistir sobre la lealtad al Estado y al partido, así como la influencia de las escuelas, los lugares de trabajo y otras dependencias burocráticas, han minado de algún modo este valor, pilar del confucianismo, y,

${ }^{39}$ Zhang Nana, "Social Networks and Women's Rural-urban Migration in Contemporary China”, Labour, Capital and Society, vol. 39, núm. 2, 2006, p. 106.

${ }^{40}$ Chan Jenny Wai-Ling, "Chinese Women Workers Organize in the Export Zones”, New Labor Forum, vol. 15, núm. 1, 2006, pp. 19-27; Zhang Nana, "Social Networks and Women's...”, op. cit., p. 105.

${ }^{41}$ Zhang Nana, "Social Networks and Women's...", op. cit.

${ }^{42}$ Ibid., p. 120. 
cada día más, la diferencia generacional y la mayor independencia de las mujeres dificulta la comunicación entre padres e hijos. En cuanto a las redes de apoyo, sigue funcionando el sistema tradicional del guanxi, por el cual, al apelar a las personas adecuadas, se intercambian servicios y se resuelven problemas en ámbitos de empleo, migración, salud y vivienda.

Dirección institucional de la autora:

Centro de Estudios de Asia y África

El Colegio de México

Camino al Ajusco 20

Pedregal de Santa Teresa

10740, México, D.F.

\botton@colmex.mx

\section{Bibliografía}

BotTon, Flora, "Cambio social y cultura", en Romer Cornejo (coord.), China: radiografía de una potencia en ascenso, México, El Colegio de México, 2008, pp. 121-206.

BotTon, Flora, "Familia y cambio social", Revista de Occidente, núm. 172, septiembre de 1995, pp. 51-64.

Bотton, Flora, “¿Qué hacer con los viejos? El problema del envejecimiento en China”, Estudios de Asia y África, vol. XLVIII (2), núm. 148, mayo-agosto de 2012, pp. 219-236.

BotTon, Flora y Romer Cornejo, "Cambio y tradición en la familia china contemporánea”, Revista de la Universidad Nacional Autónoma de México, vol. XLv, núm. 474, julio de 1990, pp. 33-37.

BotTon, Flora y Romer Cornejo, "La política de un solo hijo en China", Estudios Demográficos y Urbanos, vol. 4, núm. 2(11), mayo-agosto de 1989, pp. 343-376.

BotTon, Flora y Romer Cornejo, "Vivienda y familia en la China urbana contemporánea”, Estudios Demográficos y Urbanos, vol. 6, núm. 1 (16), enero-abril de 1991, pp. 187-207.

Botton, Flora y Romer Cornejo Bustamante, Bajo un mismo techo: la familia tradicional en China y su crisis, México, El Colegio de México, 1993.

Chan Darius K.-S., Theresa T.-T. Ng y Chin-Ming Hui, “Interpersonal Relationships in Rapidly Changing Chinese Societies", 
en Michael Harris Bond (ed.), Oxford Handbook of Chinese Psychology, Oxford-Nueva York, Oxford University Press, 2010, pp. 515-532.

CHAN Jenny Wai-Ling, "Chinese Women Workers Organize in the Export Zones”, New Labor Forum, vol. 15, núm. 1, 2006, pp. 19-27.

CHEN Feinan, Susan E. Short y Barbara Entwisle, "The Impact of Grandparental Proximity on Maternal Childcare in China", Population Research and Policy Revierw, vol. 19, núm. 6, diciembre de 2000, pp. 571-590.

Di Juxin y Emily Rosenbaum, "Caregiving System in Transition: An Illustration from Shanghai, China", Population Research and Policy Reviere, vol. 13, núm. 1, marzo de 1994, pp. 101-112.

Lang, Olga, Chinese Family and Society, New Haven, Yale University Press, 1946.

PAN, S. M., "A Sex Revolution in Current China”, Journal of Psychology and Human Sexuality, vol. 6, núm. 2, 1993, pp. 1-14.

PARISH, William L. y Martin King Whyte, Urban Life in Contemporary China, Chicago, The University of Chicago Press, 1984.

Riley, Nancy E., "Interwoven Lives: Parents, Marriage, and Guanxi in China", Journal of Marriage and Family, vol. 56, núm. 4, noviembre de 1994, pp. 791-803.

TANG, S. y J. Zuo, "Dating Attitudes and Behaviors of American and Chinese College Students”, Social Science Journal, vol. 37, núm. 1, 2000, pp. 67-78.

Whyte, Martin King, "The Fate of Filial Obligations in Urban China", The China Journal, núm. 38, julio de 1997, pp. 1-31.

Whyte, Martin King y William L. Parish, Village and Family in Contemporary China, Chicago, The University of Chicago Press, 1978.

Xu Anqi et al., Chinese Family Strengths and Resiliency, Documento núm. 53, Lincoln, Faculty Publications, Department of Child, Youth and Family Studies, 2007, pp. 143-164.

YaN Yunxiang, "Girl Power: Young Women and The Waning of Patriarchy in Rural North China", Ethnology, vol. 45, núm. 2, primavera de 2006, pp. 105-123.

YAN Yunxiang, "The Triumph of Conjugality: Structural Transformation of Family Relations in a Chinese Village”, Ethnology, vol. 36, núm. 3, 1997, p. 191-212.

ZHAN Heying Jenny y Rhonda J. V. Montgomery, "Gender and Elder Care in China: The Influence of Filial Piety and Structural Constraints", Gender and Society, vol. 17, núm. 2, abril de 2003, pp. 209-229. 
Zhang Jungfu y Zhong Zhao, Social-Family Network and SelfEmployment: Evidence from Temporary Rural-Urban Migrants in China, Documento de discusión núm. 5446, Bonn, Institute for the Study of Labor, enero de 2011.

ZHANG Nana, "Social Networks and Women's Rural-urban Migration in Contemporary China", Labour, Capital and Society, vol. 39, núm. 2, 2006, pp. 104-125.

Zhongguo chengshi jiating: Wu chengshijiating diaocha baogao be ziliao, Jinan, Shandong Renmin Chubanshe, 1985. 


\title{
Anexo
}

\section{Resumen de muestras en los artículos consultados}

\begin{tabular}{lcl}
\hline \multicolumn{1}{c}{ Artículo } & $\begin{array}{c}\text { Añode } \\
\text { publicación }\end{array}$ & \multicolumn{1}{c}{ Muestra } \\
\hline $\begin{array}{l}\text { Chan, Darius } \\
\text { K. S. et. al., } \\
\text { "Interpersonal }\end{array}$ & 2010 & $\begin{array}{l}\text { Muestras por encuestas a } \\
\text { estudiantes de universidad, } \\
\text { pelationships in } \\
\text { Rapidly Changing } \\
\text { Chinese }\end{array}$ \\
Societies". & $\begin{array}{l}\text { el artículo ofrece resultados de } \\
\text { distintos estudios y autores, } \\
\text { pero no explica una muestra } \\
\text { específica. }\end{array}$
\end{tabular}

\author{
Fuente \\ de la muestra
}

Hong Kong

Family Planning

Association:

2006 para las

estadísticas de

Hong Kong,

1998 para las

estadísticas de

Taiwan. Hong

Kong Census

and Statistics

Department,

2007, Marriage and

Divorce Trends in

Hong Kong, 1981

to 2006.

\begin{tabular}{|c|c|c|c|}
\hline $\begin{array}{l}\text { Chen Feinian et. } \\
\text { al., "The Impact } \\
\text { of Grandparental } \\
\text { Proximity } \\
\text { on Maternal } \\
\text { Childcare in } \\
\text { China". }\end{array}$ & 2000 & $\begin{array}{l}\text { La muestra es de } 850 \text { mujeres } \\
\text { con al menos un hijo/a de } \\
\text { menos de seis años de edad. La } \\
\text { muestra consiste en } 32 \text { barrios } \\
\text { urbanos, } 30 \text { pueblos, } 30 \text { pueblos } \\
\text { suburbanos; las entrevistas se } \\
\text { hicieron en } 3817 \text { hogares, de } \\
\text { los cuales } 2537 \text { son rurales y } \\
1280 \text { son urbanos. La muestra } \\
\text { no es representativa de toda } \\
\text { China; sí cubre provincias } \\
\text { con sustancial desarrollo } \\
\text { económico y geográfico. Se } \\
\text { incluyen provincias ricas y } \\
\text { pobres, de costa, del centro y } \\
\text { montañosas: realizada en ocho } \\
\text { provincias (Guangxi, Ghizhou, } \\
\text { Henan, Hubei, Hunan, Jiangsu, } \\
\text { Liaoning y Shandong). }\end{array}$ & $\begin{array}{l}\text { Datos de la } \\
\text { Encuesta de Salud } \\
\text { y Nutrición en } \\
\text { China de } 1991 .\end{array}$ \\
\hline $\begin{array}{l}\text { Di Junxin } \\
\text { y Emily } \\
\text { Rosenbaum, } \\
\text { "Caregiving } \\
\text { System in }\end{array}$ & 1994 & $\begin{array}{l}\text { Los autores no utilizan } \\
\text { una muestra en específico, } \\
\text { sino datos de distintos estudios } \\
\text { y autores, y base de datos de } \\
\text { Shanghái. }\end{array}$ & $\begin{array}{l}\text { Shanghai } \\
\text { Satistical Bureau, } \\
\text { Shanghai } 1900 \\
\text { Annual Review } \\
\text { of Statistics. }\end{array}$ \\
\hline
\end{tabular}




\section{Anexo (continuación)}

\begin{tabular}{|c|c|c|c|}
\hline Artículo & $\begin{array}{c}\text { Año de } \\
\text { publicación }\end{array}$ & Muestra & $\begin{array}{c}\text { Fuente } \\
\text { de la muestra }\end{array}$ \\
\hline
\end{tabular}

Transition-An

Illustration from

Shanghai, China".

\begin{tabular}{|c|c|c|c|}
\hline $\begin{array}{l}\text { Riley, Nancy } \\
\text { E., "Interwoven } \\
\text { Lives: Parents, } \\
\text { Marriage and } \\
\text { Guanxi in } \\
\text { China". }\end{array}$ & 1994 & $\begin{array}{l}\text { Muestra de } 5000 \text { encuestadas } \\
\text { de las provincias de Liaoning, } \\
\text { Shangdong, Beijing, } \\
\text { Guangdong, Guizhou y } \\
\text { Gangsu. Todas las encuestadas } \\
\text { son mujeres que siempre han } \\
\text { estado casadas en el mismo } \\
\text { matrimonio, con entre } 15 \text { y } 50 \\
\text { años de edad. Adicionalmente, } \\
\text { se utiliza información de grupos } \\
\text { focales recolectada en Beijing en } \\
\text { 1986-1987 con entrevistas a } 23 \\
\text { mujeres y datos cuantitativos de } \\
700 \text { mujeres solteras y casadas } \\
\text { (de entre } 20 \text { y } 35 \text { años de edad) } \\
\text { con un cuestionario estándar. }\end{array}$ & $\begin{array}{l}\text { Fertility } \\
\text { Survey, Phase } \\
\text { II, información } \\
\text { recolectada entre } \\
1986 \text { y } 1987 .\end{array}$ \\
\hline
\end{tabular}

Whyte, Martin

King, "The

Fate of Filial

Obligations in

Urban China”.
1997

Únicamente se reportan los resultados de 731 pares de padres-hijos. Muestra de 1002 residentes de los distritos urbanos de Baoding, mayores de 50 años de edad; fueron entrevistados sobre su estatus, experiencias familiares y relaciones intergeneracionales. De la muestra, se encuestó a 753 hijos. El cuestionario incluye preguntas sobre historia personal, experiencias familiares, etcétera. La pregunta central era si los habitantes de Baoding honran sus obligaciones y apoyan a sus padres o si se resisten a sus obligaciones.
Proyecto entre la Universidad de Michigan, la Universidad de Beijing y el China Research Center of Aging. Datos de 1994.
Xu Anqi et. al., Chinese Family
No se utiliza una muestra en particular, sino que el artículo
Utilizan distintos estudios basados 


\section{Anexo (continuación)}

\begin{tabular}{lll}
\hline \multicolumn{1}{c}{ Artículo } & $\begin{array}{c}\text { Añode } \\
\text { publicación }\end{array}$ & \multicolumn{1}{c}{$\begin{array}{c}\text { Fuente } \\
\text { de la muestra }\end{array}$} \\
\hline $\begin{array}{l}\text { Strengths and } \\
\text { Resiliency. }\end{array}$ & Muestra & $\begin{array}{l}\text { en datos del } \\
\text { distintos estudios y artículos. }\end{array}$ \\
& $\begin{array}{l}\text { Chinese Statistics } \\
\text { Bureau. }\end{array}$ \\
\hline
\end{tabular}

Yan Yungxiang,

"The Triumph

of Conjugality.

Structural

Tansformation of

Family Relations

in a Chinese

Village”.
1997

Datos de información

recolectados en Xiajia, en la provincia de Heilongjiang, en 1989, 1991, 1993 y 1994. Se hace una comparación entre los datos de 1980 y la encuesta realizada en 1991, en Xiajia, donde se encuestó a 1469 personas pertenecientes a 276 familias; 11 años después eran 1542 personas en 368 familias. En una década, el pueblo de Xiajia se incrementó en 73 personas y 92 nuevas familias. Lo que quiere decir que el número de hogares aumentó más rápido que el crecimiento poblacional.
Encuesta y datos del State Statistic Bureau.
Encuesta y datos del State Statistic Bureau.
"Girl Power:

Young Women and the Waning of Patriarchy in Rural North China”.

\section{6}

Entrevistas con mujeres jóvenes rurales de entre $15 \mathrm{y}$ 24 años. Datos e información recolectados en Xiajia, en la provincia de Heilongjiang, en 1989, 1991 (muestra de 36 nuevos matrimonios), 1993 (muestra de 78 matrimonios), 1994 (muestra de 49 nuevos matrimonios), 1997, 1998 y 1999.
Zhan Heyin Jenny y Rhonda J. V. Montgomery, "Gender and Elder Care in China: The Influence of Filial Piety and Structural Constraints".
Se utiliza una muestra de 110

Entrevistas familias en las cuales la entrevista es con la persona que atiende primordialmente las necesidades de cuidado de la familia y que tiene a su cargo a adultos mayores (caregivers) en zonas urbanas. Las entrevistas se hicieron en el otoño de 1997-1998. 
Anexo (conclusión)

\begin{tabular}{|c|c|c|c|}
\hline Artículo & $\begin{array}{c}\text { Año de } \\
\text { publicación }\end{array}$ & Muestra & $\begin{array}{c}\text { Fuente } \\
\text { de la muestra }\end{array}$ \\
\hline $\begin{array}{l}\text { Zhang Jungfu } \\
\text { y Zhong Zhao, } \\
\text { Social-Family } \\
\text { Network and Self } \\
\text { Employment: } \\
\text { Evidence from } \\
\text { Temporary Rural- } \\
\text { Urban Migrants in } \\
\text { China. }\end{array}$ & 2011 & $\begin{array}{l}\text { Utilizan datos de los migrantes } \\
\text { de trabajo temporal de zonas } \\
\text { rurales a urbanas en China. } \\
\text { Miden las redes sociales y } \\
\text { familiares de migrantes con el } \\
\text { número de parientes y amigos } \\
\text { que el migrante visitó durante el } \\
\text { Festival de Primavera anterior. } \\
\text { Se utiliza una encuesta única } \\
\text { para formar una base de datos } \\
\text { de la población migrante rural- } \\
\text { urbana en China e Indonesia } \\
\text { (RUMiCI). Esta encuesta } \\
\text { única fue construida por un } \\
\text { equipo de investigadores } \\
\text { (Australia, Indonesia y China) } \\
\text { y se realiza a lo largo de } \\
\text { cinco años comenzando en } \\
\text { 2008. En } 2008 \text { se tomó una } \\
\text { muestra representativa de } 8000 \\
\text { hogares rurales, } 5000 \text { hogares } \\
\text { rural-urbanos y } 5000 \text { hogares } \\
\text { urbanos. El análisis de datos } \\
\text { se concentra en población } \\
\text { migrante con el permiso } \\
\text { bukou y se encuesta de forma } \\
\text { aleatoria en } 15 \text { ciudades, de las } \\
\text { cuales ocho son en regiones } \\
\text { de costa (Shanghái, Nanjing, } \\
\text { Wuxi, Hangzhou, Ningbo, } \\
\text { Guangzhou, Shenzhen y } \\
\text { Dongguan); cinco en regiones } \\
\text { centrales (Zhengzhou, } \\
\text { Luoyang, Hefei, Bengbu y } \\
\text { Wuhan), y dos en regiones del } \\
\text { oeste (Chengdu y Chonging). } \\
\text { El estudio contiene más de } \\
700 \text { variables de características } \\
\text { personales, familiares y } \\
\text { profesionales de los trabajadores } \\
\text { migrantes. La muestra resultó de } \\
4442 \text { hogares (observaciones). }\end{array}$ & $\begin{array}{l}\text { Utilizan datos del } \\
\text { National Bureau } \\
\text { of Statistics } \\
2008 \text {, donde hay } \\
\text { un registro de } \\
225 \text { millones } \\
\text { de migrantes } \\
\text { rural-urbanos en } \\
\text { China. }\end{array}$ \\
\hline
\end{tabular}

